\title{
A Search for a Charged Lepton Flavour Violating Process; Muon to Electron Conversion in COMET
}

\author{
Yuki Fujii*广 \\ Faculty of Sciene, Monash University \\ E-mail: yuki.fujii@monash.edu
}

The COMET experiment is designed to search for a muon to electron $(\mu-e)$ conversion without associating neutrinos, where the lepton flavour is violated at the charged lepton sector. The charged lepton flavour violating processes (CLFV) are forbidden in the Standard Model, while many plausible models beyond the SM (BSM) predict the detectable rates of CLFV processes. Therefore the CLFV searches are strong tools to probe the BSM. If any of CLFV process is discovered, it would be the clear evidence of BSM. The aim of the COMET experiment is to search for the $\mu$-e conversion with a four orders of magnitude better sensitivity than the present upper limit. The experiment adopts the staging approach and the first stage of COMET is under construction at J-PARC/Japan, to search for the $\mu$-e conversion with the intermediate sensitivity, $3 \times 10^{-15}$ in the early 2020 s, followed by the Phase-II experiment to achieve the 100 times further improved sensitivity. We recently measured the quality of proton beam at J-PARC and it satisfied our exceedingly strict requirement. Preparations for the beam-line, detectors and electronics are intensively progressing on schedule. In addition, we recently started further optimisations for Phase-II experiment to improve the target sensitivity by factor 2-10 from the current baseline design, $3 \times 10^{-17}$. In this paper, the current status of the COMET experiment, mainly focusing on the recent highlights those of above mentioned are summarised together with future prospects.

European Physical Society Conference on High Energy Physics - EPS-HEP2019 -

10-17 July, 2019

Ghent, Belgium

\footnotetext{
*Speaker.

${ }^{\dagger}$ On behalf of the COMET collaboration
} 


\section{Introduction}

The Standard Model of particle physics (SM) has withstood various experimental tests for several decades. Although that, the existence of new physics Beyond the SM (BSM) is required to account for the true identity of dark matter, tiny mass of neutrinos, matter-antimatter asymmetry in the universe, etc. A COMET (COherent Muon to Electron Transition) experiment is exploring the BSM by searching for the neutrinoless muon-to-electron $(\mu-e)$ conversion which violates the conservation law of charged lepton flavours in the SM. Branching ratios of charged lepton flavour violating processes (CLFV) are strongly suppressed in the SM due to the tiny neutrino mass. In contrast, they can be significantly enhanced up around $10^{-14}$ by introducing additional (generally heavy) mediating particle(s) in many plausible BSMs, for example Supersymmetry. If any of CLFV process is discovered, it would be a clear evidence of BSM. Furthermore, CLFV searches are indirectly sensitive to the energy scale up to $\sim 10,000 \mathrm{TeV}$ which is directly inaccessible by any existing particle colliders. The COMET experiment aims to search for the $\mu$-e conversion with 100 and 10,000 times better sensitivities than the present upper limit, $7 \times 10^{-13}$ given by SINDRUM II [1], in its Phase-I and Phase-II, respectively [2]. The experiment will be done at J-PARC (Japan Proton Accelerator Research Complex) to obtain more than $10^{18}$ of muons by employing a high intensity bunched proton beam. This paper reviews the experimental overview of COMET, the status of beam-line and each detector developments together with the recent highlights.

\section{Signal and Backgrounds}

A signal topology of the $\mu-e$ conversion is as simple as follows. Once a negative muon loses most of its kinetic energy inside the materials, it is captured by an atom and forms muonic atom. If a $\mu$-e conversion occurs, only an electron is emitted with a mono-energy of $E_{\mu-e}=M_{\mu}-B_{\mu} \sim$ $105 \mathrm{MeV}$ with a lifetime of $864 \mathrm{~ns}$ in case of an aluminium nucleus. Here $M_{\mu}$ is the muon mass and $B_{\mu}$ is the binding energy of $1 s$-state muonic atom. In the SM, a muon decays as $\mu^{-} \rightarrow e^{-} \bar{v}_{e} v_{\mu}$ in an orbit (DIO) or is captured by a nucleus. The energy spectrum of DIO electrons peaks around $M_{\mu} / 2 \approx 52.8 \mathrm{MeV}$, however, it also has a tiny tail component up to $105 \mathrm{MeV}$ due to the nuclear recoil. Hence, the momentum of an electron should be measured with a few $100 \mathrm{keV} / \mathrm{c}$ precision to suppress the DIO background. Another main background is induced by the prompt beam since electrons with around $100 \mathrm{MeV} / \mathrm{c}$ can be generated by in-flight decay of pions and muons. Due to the short lifetime of pions, most of secondary particles arrive the detector region within several $100 \mathrm{~ns}$ after the main proton beam arrival. These backgrounds can therefore be strongly suppressed by setting the measurement time window delayed by $500-700 \mathrm{~ns}$ with respective to the beam arrival time with the bunched beam structure. In this scheme, the high purity bunched beam is essential. The factor called extinction ( $\equiv$ (Number of residual protons between consecutive bunches)/(Number of protons in a bunch)) must be smaller than $10^{-10}$ to achieve the sensitivity better than $10^{-16}$.

\section{COMET Experiment}

Experimental Overview: In order to achieve the single event sensitivity (SES) better than an $O\left(10^{-14}\right)$, following requirements must be fulfilled; (1) accumulate more than $10^{18}$ of stopped 
muons, (2) make background level lower than the SES, and (3) maintain a modest data acquisition (DAQ) rate under the high intensity environment. The COMET experiment is designed to satisfy those requirements as shown in Fig. 1 and 2 for Phase-I and Phase-II, respectively. In the J-PARC main ring (MR), $2.4 \times 10^{12}\left(4.2 \times 10^{13}\right) \mathrm{s}^{-1}$ of bunched protons are accelerated up to $8 \mathrm{GeV}$ with a beam power of 3.2(56) kW for Phase-I(Phase-II). All protons are delivered to the COMET experimental area and impinged into the graphite(tungsten) production target located at the centre of the capture solenoid in Phase-I(Phase-II). A lot of pions and muons are generated through hadronic interactions and captured by the solenoidal field of $5 \mathrm{~T}$. Charged particles with momenta less than $100 \mathrm{MeV} / \mathrm{c}$ travel to the transport solenoid effectively owing to the gradually decreasing magnetic field. The $\mathrm{C}$-shaped solenoid is designed to have a maximum transportation efficiency for negative charged particles with low momenta toward the detector solenoid and pions that could cause the severe background are strongly suppressed during the transportation due to their short lifetime. In Phase-I, we will conduct two important measurements using different detector systems. A Cylindrical Detector (CyDet) will perform the physics measurement with a SES of $O\left(10^{-15}\right)$ which improves the current upper limit by factor 100. A detector system called StrECAL, which also corresponds to the prototype Phase-II detector, will directly measure the beam properties at the $90^{\circ}$ bend of transport solenoid to obtain the precise background information. This will enable the further optimisations on the Phase-II experimental design.
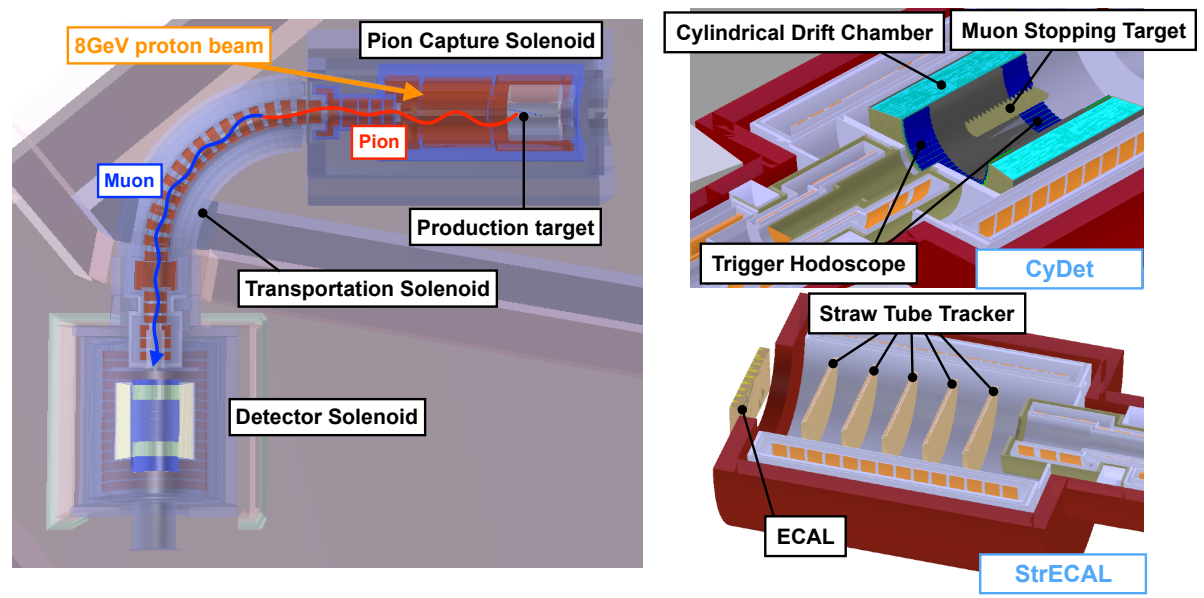

Figure 1: An overview of COMET Phase-I. CyDet will search for the $\mu-e$ conversion while StrECAL will measure the beam profile as Phase-II prototype detectors.

Beam-line: COMET requires the dedicated beam-line which delivers the $8 \mathrm{GeV}$ proton beam with a bunched structure to the experimental area in the hadron experimental facility. The design of the beam-line has been already fixed and the construction is ongoing. In 2018, we performed the extinction measurement using a $8 \mathrm{GeV}$ bunched beam both at the abort beam-line in MR and the secondary beam-line called K1.8 at the hadron facility. The operation of the $8 \mathrm{GeV}$ bunched proton beam with an interval of $1.17 \mu \mathrm{s}$ has been performed in the measurement. Moreover, we confirmed that the quality of beam are consistent with expectations and the extinction factor was measured to be better than $6 \times 10^{-11}$ which satisfies the requirement [3].

CyDet: As shown in right top of Fig. 1, The CyDet consists of a cylindrical drift chamber (CDC) and an array of scintillatoion counters and Cherenkov radiators called CyDet trigger hodoscope $(\mathrm{CTH})$. The $\mathrm{CDC}$ has $\approx 5,000$ sensitive wires in 20 layers with alternate stereo angles in 


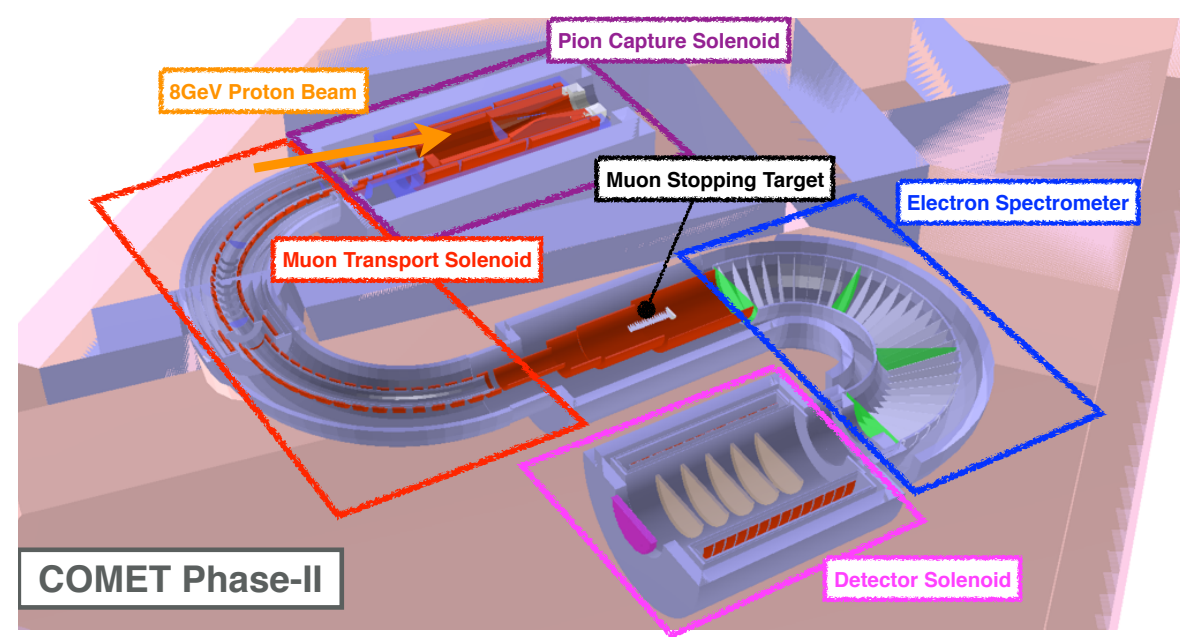

Figure 2: An overview of COMET Phase-II. Longer Muon transport solenoid and an additional electron spectrometer will further suppress backgrounds.

order to reconstruct the 3D hit positions and momenta with helium based gas mixture (He:iso$\mathrm{C}_{5} \mathrm{H}_{10}=90: 10$ ). The construction of CDC was completed in 2015 and the spacial resolution of $170 \mu \mathrm{m}$, which satisfies the requirement, has been confirmed by using cosmic-rays [4]. The CDC will also contribute to suppress the trigger rate by combining the innovative machine learning technique and costum FPGA-based trigger electronics [5]. The CTH will measure the timing of signal electrons with $1 \mathrm{~ns}$ precision and provide the primary trigger signal with a sufficiently low trigger rate of less than $\sim 100 \mathrm{kHz}$. Both scintillation counters and Cherenkov radiators are segmented by 48 and cylindrically aligned, located at both ends of CDC. A low trigger rate will be realised by taking the coincidence of neighbouring and overlapped 2 scintillation counters and 2 Cherenkov radiators. The prototype of CTH has been built and the sufficient performance has been confirmed.

StrECAL: Another detector system called StrECAL (see also right bottom of Fig. 1) consisting of a thin straw tube tracker and an electron calorimeter (ECAL) will perform the Phase-I beam measurement and Phase-II physics measurement. All detectors will be operated inside the vacuum because the momentum resolution of $105 \mathrm{MeV} / \mathrm{c}$ electrons are dominated by the multiple scattering. The straw tube tracker is composed of 5-7 stations and each station has two straw tube layers perpendicularly aligned. Each straw tube has only 20(12.5) $\mu \mathrm{m}$ thickness aluminized mylar wall with 10(5) mm diameter filled with Argon:Ethane evenly mixed gas to minimise the material budget for Phase-I(Phase-II). All the Phase-I straw tubes have been already produced and the target spacial resolution of $150 \mu \mathrm{m}$ has been achieved in the test beam measurement by using the full scale prototype [6]. The construction of the detector has begun accordingly. In addition, we recently succeeded to manufacture the $12.5 \mu \mathrm{m}$ thickness straw tubes with $5 \mathrm{~mm}$ of diameter, which will be used in the Phase-II physics measurement. Further studies are ongoing to assemble this ultra thin straw tubes as a tracking detector. The ECAL will provide the energy and timing information of incoming particles together with the primary trigger decision. It consists of $\approx 2,000$ of LYSO crystals, $2 \mathrm{~mm} \times 2 \mathrm{~mm} \times 120 \mathrm{~mm}$ each, that will give the energy resolution better than $5 \%$ and timing resolution of $0.5 \mathrm{~ns}$ for $105 \mathrm{MeV}$ electrons. A part of crystals were already purchased and the design of the detector has been almost fixed based on the prototype study [7]. 
Sensitivity and Background: The branching ratio of the $\mu-e$ conversion is given as

$$
B\left(\mu^{-}+\mathrm{Al} \rightarrow e^{-}+\mathrm{Al}\right)=\frac{1}{N_{\mu} \cdot B_{c a p} \cdot f_{\text {gnd }} \cdot A_{\mu-e}},
$$

here $N_{\mu}$ is the number of stopped muons, $B_{\text {cap }}=0.61$ is the branching ratio for muon nuclear capture in aluminium, $f_{\text {gnd }}$ is a model dependent fraction of muonic atom in a ground state set to 0.9 here, and $A_{\mu-e}$ is a total acceptance determined by the experiment. Thus, the SES can be calculated when $N_{\mu}$ and $A_{\mu-e}$ are given. The background is estimated by generating the large amount of simulations with correctly applied physics models.

In COMET, we are developing our own software package called ICEDUST based on Geant 4 and ROOT. ICEDUST contains the custom physics models especially for stopping muons based on the theory and measurements. The SES and number of backgrounds were estimated by using ICEDUST for both phases of experiment and results are shown in Table 1 and 2. In Phase-II, the beam intensity can be 18 times higher than in Phase-I owing to the longer transport solenoid and an additional electron spectrometer, that reduce the backgrounds and hit rate in detectors. The production target replacement from graphite to tungsten will lead to three times higher stopping muons per $\mathrm{POT}^{1}$ at the aluminium target disks. Furthermore, the total signal acceptance will be improved by $40 \%$ mainly due to the larger geometrical acceptance than in Phase-I, and the momentum resolution will be improved by using the $12.5 \mu \mathrm{m}$ thickness straw tube tracker inside the vacuum. Those improvements enables to apply the tighter momentum window to reduce the contamination of DIO background. By taking into account Eq. 3.1 and numbers in Table 1, experimental sensitivities are expected to be $S E S_{\text {Phase-I }}=2.7 \times 10^{-15}$ and $S E S_{\text {Phase-II }}=3.5 \times 10^{-17}$ by assuming the 150 days of total DAQ time. The calculated total backgrounds are sufficiently low in both phases as shown in Table 2.

Recently, we revisited the SES estimation using our simulation for Phase-II, and it was found that the number stopping muons can be more than doubled by the current estimation by modifying the geometry of the production target and the geometry and magnetic field of the transportation solenoid. Besides, further optimisations on the stopping target geometry and surrounding magnetic field gives higher muon stopping efficiency. Putting them together, the stopping muon rate can be 2-5 times larger with the same power of proton beam. By adding another optimisation of the electron spectrometer, which selects $105 \mathrm{MeV}$ electrons efficiently compared to the low momentum particles, the signal acceptance can be doubled at most. Hence, there is a big room to improve the $S E S_{\text {Phase-II }}$ by factor $2-10$ if the background level can be kept low enough.

\section{Conclusion}

A large branching ratio of the $\mu-e$ conversion is predicted in many BSMs whilst it is almost undetectable in the SM. Thus, the $\mu$-e conversion search is one of the promising probes of new physics. The COMET experiment searches for the $\mu$-e conversion with unprecedented SES of $O\left(10^{-15}\right)$ and $O\left(10^{-17}\right)$ in its Phase-I and Phase-II, respectively. The quality of primary proton beam and its extinction factor have been measured to be in an extremely good level. All detectors

\footnotetext{
${ }^{1}$ A Proton On the production Target.
} 
Table 1: Summary of the sensitivity calculations in Phase-I and Phase-II.

\begin{tabular}{lll}
\hline \hline Contributions & Phase-I & Phase-II \\
\hline Beam power $(\mathrm{kW})$ & 3.2 & 56 \\
Muon stopping rate $\left(\times 10^{-4} \mu^{-} /\right.$POT $)$ & 5.0 & 16 \\
\hline Total stopped muons $\left(\times 10^{16} /(150\right.$ days $\left.)\right)$ & 1.6 & 91.3 \\
\hline Geometrycal acceptance & 0.18 & 0.22 \\
Timing window acceptance & 0.3 & 0.53 \\
Trigger+DAQ efficiency & 0.9 & 0.9 \\
Reconstruction efficiency & 0.99 & 0.9 \\
Offline event selection efficiency & 0.84 & 0.63 \\
\hline Total signal acceptance & 0.041 & 0.057 \\
\hline
\end{tabular}

Table 2: Summary of the background rates calculated for Phase-I and Phase-II.

\begin{tabular}{|c|c|c|c|}
\hline \multirow[t]{2}{*}{ Type } & \multirow[t]{2}{*}{ Source } & \multicolumn{2}{|c|}{ Expected events } \\
\hline & & Phase-I & Phase-II \\
\hline \multirow[t]{2}{*}{ Intrinsic } & DIO & 0.01 & 0.068 \\
\hline & Radiative muon capture & $1.9 \times 10^{-3}$ & negligible \\
\hline \multirow[t]{3}{*}{ Prompt } & Radiative pion capture & $2.8 \times 10^{-3}$ & $1.2 \times 10^{-3}$ \\
\hline & Beam particles & $<3.8 \times 10^{-3}$ & $1.9 \times 10^{-3}$ \\
\hline & $\bar{p}$ induced $\mathrm{BG}$ & negligible & negligible \\
\hline \multirow[t]{3}{*}{ Deleyed } & Beam & negligible & negligible \\
\hline & Radiative pion capture & negligible & $1.0 \times 10^{-3}$ \\
\hline & $\bar{p}$ induced $\mathrm{BG}$ & $1.2 \times 10^{-3}$ & 0.30 \\
\hline Others & Cosmic ray induced BG & $<0.01$ & 0.29 \\
\hline Total & & 0.032 & 0.66 \\
\hline
\end{tabular}

are being prepared on schedule to start the measurement immediately after the completion of the dedicated beam-line expected in early 2020s. The sensitivity and background in each stage are evaluated with realistic geometries, magnetic field and reliable physics models. The results show that our target sensitivities are achievable with sufficiently low background levels, and indicate the further possible improvement in Phase-II.

\section{Acknowledgements}

We acknowledge support from JSPS, Japan; Belarus; NSFC, China; IHEP, China; IN2P3-CNRS, France; CC-IN2P3, France; SRNSF, Georgia;JINR; IBS, Korea; RFBR, Russia; STFC, United Kingdom; and Royal Society, United Kingdom.

\section{References}

[1] W. H. Bertl et.al.[SINDRUM-II Collaboration], "A Search for muon to electron conversion in muonic gold”, Eur. Phys. J. C47, pp.337-346 (2006).

[2] G. Adamov et.al.[COMET Collaboration], “COMET Phase-I Technical Design Report”, arXiv:1812.09018 (2018). 
[3] H. Nishiguchi et.al., "Extinction Measurement of J-PARC MR with $8 \mathrm{GeV}$ Proton Beam for the New Muon-to-Electron Conversion Search Experiment - COMET", JACoW IPAC2019, Conference Proceedings, pp.4372-4375, doi:10.18429/JACoW-IPAC2019-FRXXPLS2 (2019).

[4] M. Moritsu et.al., "Construction and performance tests of the COMET CDC", PoS ICHEP2018, Conference Proceedings, 538, doi:10.22323/1.340.0538 (2019).

[5] Y. Nakazawa et.al., "Fast Online Trigger using FPGA-based Event Classification for COMET Phase-I”, PoS EPS-HEP2019 (this conference, not published yet).

[6] H. Nishiguchi et.al., "Development of an extremely thin-wall straw tracker operational in vacuum-The COMET straw tracker system”, Nucl. Instrum. Methods A, 845, pp.269-272, doi:10.1016/j.nima.2016.06.082 (2017).

[7] K. Oishi, "Development of Electromagnetic Calorimeter Using LYSO Crystals for the COMET Experiment at J-PARC”, PoS EPS-HEP2017, Conference Proceedings, 800, doi:10.22323/1.314.0800 (2018). 